\title{
Material erosion and deposition on the divertor of W7-X
}

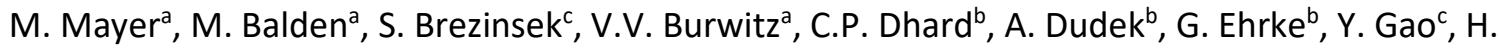 \\ Greuner $^{a}$, R. Guimarães ${ }^{d}$, P. Hiret ${ }^{a}$, S. Klose ${ }^{b}$, R. König ${ }^{b}$, M. Krause ${ }^{b}$, R. Laube $^{b}$, M. Laux ${ }^{b}$, D. Naujoks ${ }^{b}$, R. \\ $\mathrm{Neu}^{\mathrm{a}}$, J. Oelmann ${ }^{c}$, C. Ruset ${ }^{\mathrm{e}}$, T.S. Silva ${ }^{\mathrm{d}}, \mathrm{R}^{\mathrm{R}} \mathrm{Yi}^{\mathrm{c}}$, D. Zhao ${ }^{\mathrm{c}}$, and W7-X Team \\ ${ }^{a}$ Max-Planck-Institut für Plasmaphysik, Garching, Germany \\ ${ }^{b}$ Max-Planck-Institut für Plasmaphysik, Greifswald, Germany \\ ${ }^{c}$ Forschungszentrum Jülich GmbH, Jülich, Germany \\ ${ }^{d}$ University of São Paulo, São Paulo, Brazil \\ ${ }^{e}$ National Institute for Laser, Plasma and Radiation Physics, Bucharest, Romania
}

\begin{abstract}
The net erosion and deposition pattern of carbon from the Test Divertor Unit (TDU) of the stellarator W7-X was determined. Special target elements with marker layers consisting of about $300 \mathrm{~nm}$ molybdenum and 5-10 $\mu \mathrm{m}$ carbon on top were used during the operation phase OP 1.2a. The thicknesses of the marker layers were determined by elastic backscattering spectrometry (EBS) using $2.5 \mathrm{MeV}$ protons before and after plasma exposure and laser-induced breakdown spectroscopy (LIBS) on selected target elements after exposure. Scanning electron microscopy (SEM) was used for investigating the surface morphology before and after exposure. Massive erosion of up to $20 \mu \mathrm{m}$ carbon was observed at the strike line, in total $48 \pm 14 \mathrm{~g}$ carbon were eroded from the 10 TDUs. The erosion was laterally nonuniform on the micro-scale. Strongly eroded surfaces were considerably smoother as compared to the original material. Only very little deposition of carbon is observed on the TDU: This means that the TDU is a large net erosion source.
\end{abstract}

\section{Introduction}

The Wendelstein 7-X (W7-X) experiment $[1,2,3,4]$ is an advanced stellarator with a plasma volume of $30 \mathrm{~m}^{3}$, a superconducting coil system operating at a magnetic field of $2.5 \mathrm{~T}$, and steady-state heating capabilities of up to $8 \mathrm{MW}$. It is in operation since 2015. For the first operational phase (OP 1.1) only a minimal set of five discrete inboard graphite limiters was used as plasma-facing components (PFCs). For the second operational phase (OP 1.2) an adiabatically loaded divertor, the so-called Test Divertor Unit (TDU) $[5,6]$, baffles, an inner heat shield, wall panels and toroidal as well as poloidal closures were installed. Except for the wall and pumping gap panels and poloidal closures all PFCs are made of finegrain graphite. The TDU provides a larger area of plasma-facing surface and allows an improved particle exhaust as compared to limiter operation, thus allowing much higher injected heating energies per plasma pulse and a better separation between the plasma-wall interacting region and the core plasma. The TDU implements the so-called island divertor concept [7], where intrinsic chains of islands at the 
plasma edge provide multiple x-points and the plasma intersects the divertor plates at some distance from the closed flux surfaces. The successful application of this concept was already demonstrated at the antecessor experiment W7-AS [8]. The TDU has the same plasma-facing contour as the water-cooled steady-state divertor equipped with carbon-fiber-composite PFCs that is currently being installed and will be in operation in the early 2020's.

The investigation of plasma-wall interactions (PWI) in W7-X is challenging due to the three-dimensional twisted plasma geometry and the consequently curved shape of all PFCs, the high divertor heat loads (up to $10 \mathrm{MW} / \mathrm{m}^{2}$ ) and the large size of the components. For PWI investigations 18 exchangeable divertor target elements were coated with C/Mo marker layers and exposed during the operational phase OP 1.2a in 2017; the whole set of target elements was exchanged between the operational phases OP 1.2a and OP 1.2b. This paper describes the details of the experimental setup and the erosion/deposition pattern observed in OP 1.2a.

\section{Experimental}

\subsection{Plasma operation and wall conditioning}

The operational phase OP 1.2a lasted from August to December 2017. In total 1248 plasma discharges were performed, see Table 1, with a total plasma time of $3776 \mathrm{~s}$. Most discharges $(65.7 \%$ of the plasma time) were in Standard configuration, the remaining discharges were in High lota or High Mirror configuration or their derivatives. Low lota discharges were not performed in OP 1.2a. $72.2 \%$ of the plasma time were nominally He fuelled discharges. However, even in the case of He fuelling the plasmas were usually mixed $\mathrm{H}+\mathrm{He}$ discharges with varying (and often only minority) He content. Deuterium discharges were not performed in OP 1.2a.

The Standard Configuration has its strike line on the horizontal target modules TM1h to TM4h and the vertical target modules TM1v to TM3v (see Fig. 2), the High Mirror configuration has the strike line on TM1v to TM3v and TM3h to TM4h. The High lota configuration has the strike line in the high iota part of the divertor outside of the investigated area of this study. See e.g. [6] for details of the different configurations. Peak divertor heat loads of up to $8 \mathrm{MW} / \mathrm{m}^{2}$ and strike line widths of up to $11 \mathrm{~cm}$ were observed using infrared thermography, the plasma wetted divertor area increased about linearly with ECRH heating power up to values of $1.5 \mathrm{~m}^{2}$ at $5 \mathrm{MW}$ [6]. The divertor bulk target temperature increased throughout the experimental day, seldomly up to $400{ }^{\circ} \mathrm{C}[6]$. The surface temperature at the strike line could reach temperatures up to $800^{\circ} \mathrm{C}$ [9]. The electron temperature in the scrape-off layer was $50-$ $100 \mathrm{eV}$ with electron densities of $2-6 \times 10^{18} \mathrm{~m}^{-3}[10,11]$.

Glow discharge cleaning (GDC) was regularly performed for wall conditioning. 2:58 $\mathrm{h}$ of $\mathrm{H}_{2}$-GDC and 0:36 $\mathrm{h}$ of He-GDC were performed prior to the first plasma. 13:46 $\mathrm{h}$ of $\mathrm{H}_{2}-\mathrm{GDC}$ and 14:48 $\mathrm{h}$ of He-GDC were performed during the campaign. There were no boronizations applied in OP 1.2a.

\subsection{Exchangeable PWI target elements in the TDU}


Special exchangeable PWI target elements for erosion/deposition investigations were developed [12], a schematic representation is shown in Fig. 1. Each PWI target element consists of a fixed bar and a removable rider frame with mounted graphite tiles. The bar has three bayonet couplings allowing easy slide-in of the rider frame which is finally fixed with a single screw. This construction allows easy exchange of the rider frame with graphite tiles inside the machine. The bar and the rider frame were manufactured from the high-temperature molybdenum-based alloy TZM, the graphite tiles were manufactures from isostatically pressed fine grain graphite SGL SIGRAFINE R6710. The lengths of the target elements were up to $600 \mathrm{~mm}$, the sizes of the graphite tiles were typically $75 \times 50 \times 30 \mathrm{~mm}^{3}$ with differing start and end tiles.

For qualifying the construction a test target element was exposed in the high heat flux facility GLADIS [13] at heat loads up to $8.5 \mathrm{MW} / \mathrm{m}^{2}$ for $6.2 \mathrm{~s}$. For a start temperature of $200{ }^{\circ} \mathrm{C}$ the maximum surface temperature reached $1910^{\circ} \mathrm{C}$. Plastic deformations or other problems (for example cracks) were not observed. This qualification was beyond the actually achieved values in W7-X.

W7-X has 10 TDUs of which 5 are lower TDUs (1I to 5I) and 5 upper TDUs ( $1 \mathrm{u}$ to $5 \mathrm{u}$ ), see e.g. [5,9] for a schematic representation. In total 18 PWI target elements were used, see Table 2 and Fig. 2 for a schematic representation of the locations of the elements. The position TM2h6 in the horizontal target was used in all TDUs for studying the toroidal asymmetry of the erosion/deposition pattern, additionally the positions TM1h3 and TM3h6 in the horizontal and TM1v5 and TM2v2 in the vertical targets were used in TDUs $5 \mathrm{I}$ and $5 \mathrm{u}$.

\subsection{Marker layers for erosion/deposition measurements}

Carbon/molybdenum marker layers were used for determining erosion of carbon and deposition of wall materials and impurities, e.g. $\mathrm{C}, \mathrm{O}, \mathrm{Ni}$, Fe etc. on the TDU. The marker layers were deposited as an about $2 \mathrm{~cm}$ wide stripe in the middle of the graphite tiles using the CMSII technique [14]. The markers consist of an about $200 \mathrm{~nm}$ thick Mo layer and a 5-10 $\mu \mathrm{m}$ thick C layer on top. The Mo serves as indicator for distinguishing the $\mathrm{C}$ layer from the bulk $\mathrm{C}$ of the tile.

Test marker layers were tested in GLADIS with 50 pulses at a heat load of $8 \mathrm{MW} / \mathrm{m}^{2}$ for $2.5 \mathrm{~s}$, the maximum surface temperature was $1200^{\circ} \mathrm{C}$. Optically no change of the layer was visible, scanning electron microscopy (SEM) showed small cracks in the layer probably due to different thermal expansion coefficients. Delamination of the layer was not observed.

\subsection{Analysis of the marker layers}

The marker layers were analyzed before and after exposure in W7-X using Elastic Backscattering Spectrometry (EBS) at the IPP tandem accelerator facility in the Bombardino chamber using the BesTec flange. 2.5 MeV incident protons at normal incidence and a scattering angle of $165^{\circ}$ were used, see Figs. 1 and 2 in [15] for typical spectra. A passivated implanted planar silicon (PIPS) detector with a thickness of $300 \mu \mathrm{m}$ and nominal energy resolution of about $12 \mathrm{keV}$ was used. The pre-exposure measurements were performed with a step width of $20 \mathrm{~mm}$ in order to determine variations of the initial layer thicknesses, the post-exposure measurements were made with a step width of $5 \mathrm{~mm}$. In total almost 3000 spectra were recorded. 
The measured spectra were analyzed using the program SIMNRA $[16,17]$ with SRIM [18] stopping powers and non-Rutherford SigmaCalc scattering cross-sections for ${ }^{12} \mathrm{C},{ }^{13} \mathrm{C}$ and ${ }^{16} \mathrm{O}[19]$. Surface roughness was taken into account using the simplified model from [20].

The large number of acquired spectra required a special approach. The spectra were fitted using different methods:

1. The measurements before exposure (about 500 spectra) were fitted manually.

2. All measurements were fitted using automated fits. The total computing time for all fits was about 5000 CPU hours, the fits were run in 20 parallel threads on multiple PCs.

3. An Artificial Neural Network (ANN) was trained using about 1 million simulated spectra calculated by SIMNRA $[16,17,20]$ with added artificial Poisson counting statistics. $75 \%$ of the dataset was used for training and $25 \%$ was used for validation. The ANN had 3 hidden layers and was trained to predict the amounts of carbon and molybdenum. The "Adam" solver was used, iterative learning with $\alpha=0.0001$, initial learning rate 0.001 , tolerance 0.0001 . Once trained the ANN evaluated a spectrum in less than $1 \mathrm{~s}$.

Laser-Induced Breakdown Spectroscopy (LIBS) measurements were performed in a vacuum of $10^{-5} \mathrm{~Pa}$ using a laser pulse length of $35 \mathrm{ps}$ with a repetition rate of $10 \mathrm{~Hz}$ at a wave length of $355 \mathrm{~nm}$ [21]. The laser beam spot diameter was $0.7 \mathrm{~mm}$. The plasma emission was observed with an optical fibre and collected by a compact Czerny-Turner spectrometer from Ocean Optics in the wavelength range from 350 to $800 \mathrm{~nm}$ with a resolution of about $0.2 \mathrm{~nm} / \mathrm{pixel}$. This wavelength range covers lines of hydrogen and of relevant impurities, namely $\mathrm{C}, \mathrm{N}, \mathrm{O}, \mathrm{Mo}$ as well as $\mathrm{B}$.

Scanning electron microscopy was performed with a FEI Helios nanolab 600 with focused ion beam (FIB) for cross-sectioning and energy dispersive X-ray spectroscopy (EDX) capabilities. The electron beam energy was $5 \mathrm{keV}$.

\section{Results}

\subsection{Scanning electron microscopy}

Scanning electron micrographs of unexposed marker samples are shown in Fig. 3. The micrographs were recorded with secondary electrons. A typical micrograph of the raw tile surface is shown in Fig. 3a). The surfaces of the fine-grain graphite tiles were grinded and not polished in order to have a comparable surface finish as all other graphite tiles in W7-X, the surfaces are therefore generally very rough on the $\mu \mathrm{m}$-scale. In addition to this small-scale roughness of the material large grooves due to the grinding process and deep pores are observed. The surface of the Mo/C marker layer is shown in Fig. $3 \mathrm{~b}$ ). This surface is generally smoother on the $\mu \mathrm{m}$-scale as the raw material and shows larger hemispheric structures. Fig. 3c) shows a FIB cross-section through the $\mathrm{C}$ and Mo marker coatings into the graphite substrate. The Mo layer with a thickness of 200-300 nm follows the morphology of the fine-grain graphite surface and is consequently very rough. It also penetrates into the interior of pores. 
Typical scanning electron micrographs of the Mo/C marker coating after exposure during OP 1.2a are shown in Fig. 4 for a) the high erosion area at the strike line in standard configuration, b) a medium erosion area, and c) in a remote area. The erosion-dominated areas are considerably smoother than the initial marker coating, see Fig. 3b), and also smoother than the initial raw graphite. At the strike point area of this target element, see Fig. 4 a), not only the $\mathrm{C}$ marker coating, but also the Mo layer was completely eroded. Small remnants of the Mo layer could be observed in backscattered electron (BSE) images at the bottom of pores (not shown). In the medium erosion area inhomogeneous erosion is observed: At many places the carbon marker layer is still present above the Mo layer, while at some other places the $\mathrm{C}$ marker layer was completely eroded and the Mo can be observed at the surface. No indications for delamination, either of the $\mathrm{C}$ marker layer or the Mo layer, could be found. In remote areas close to the outer baffle, see Fig. 4c), the surface shows only very small modifications. The plasmaexposed surfaces are slightly brighter than shadowed areas indicating a somewhat different secondary electron yield on plasma-exposed surfaces: This is an indication for a thin deposit. SEM images at higher magnification (not shown) confirmed the existence of a thin deposit.

\subsection{Erosion/deposition pattern}

The initial thicknesses of the Mo and C layers, as determined by EBS on target element 1u TM2h6, are shown in Fig. 5 as hollow symbols together with a schematic representation of the target element. The origin of the shown coordinate system along the target element is the gap between the first and the second tile on the pumping-gap side. The results of the pre-analysis from the ANN analysis, the manual analysis, and the automated fits agree very well. The thicknesses of the Mo and Clayers on the different tiles show some variation due to layer deposition in different coating runs, but also due to the position of each tile in the coating device during the coating process. Some tiles also show a thickness gradient of the order of $10 \%$ over the length of the tile. This variation of layer thicknesses on different tiles demonstrates the necessity of careful pre-analysis of each individual tile.

The thicknesses of the Mo and C layers, as determined by ANN analysis of the EBS spectra, are shown in Fig. 5 as solid symbols. From about $-320 \mathrm{~mm}$ to $0 \mathrm{~mm}$ the carbon marker layer has been (partly or completely) eroded. Around about $-200 \mathrm{~mm}$ both the $\mathrm{C}$ and the Mo layer have been, at least partly, eroded: This is due to the inhomogeneous erosion observed in the SEM images, see Fig. 4b) for a typical image. From about $-130 \mathrm{~mm}$ to $-50 \mathrm{~mm}$ the carbon layer was completely and the Mo layer below was partly eroded, with almost complete erosion of the Mo layer at about $-100 \mathrm{~mm}$, see Fig. 4a) for a typical representation of such an area.

The thicknesses of the $\mathrm{C}$ layer are almost identical before and after exposure in areas without direct plasma contact close to the outer baffle $(-470 \mathrm{~mm}$ to $-320 \mathrm{~mm})$. The Mo layer thicknesses show a larger scatter in these areas: This is partly due to an increased roughness of the top C layer after exposure, which results in a larger scatter of the Mo data, and partly due to an increase of the oxygen signal, which partly overlaps with the Mo signal. Except this increase of the amount of oxygen the deposition of other elements, for example Fe or $\mathrm{Ni}$, was below the detection limit.

Close to the pumping gap ( $0 \mathrm{~mm}$ to $50 \mathrm{~mm}$ ) typically a (small) increase of the amount of $\mathrm{C}$ was observed. 
The difference between the layer thicknesses before and after exposure, i.e. the net erosion of $\mathrm{C}$ and Mo, is shown in Fig. 6 for target element 1u TM2h6 (the initial layer thicknesses are shown in Fig. 5). The layer thicknesses before exposure were linearly interpolated (and extrapolated at the tile edges if necessary). The uncertainty of the Mo data points was $2.7 \times 10^{17} \mathrm{Mo}$-atoms $/ \mathrm{cm}^{2}$ and was determined by a statistical analysis of all Mo data points in remote areas where the top $C$ layer was still present: In these areas the net erosion of Mo should be zero, and the uncertainty of the net Mo erosion can be determined as deviation of the determined Mo erosion from zero. Uncertainties of the net $\mathrm{C}$ erosion were determined on target element TM1h3 in TDU 5I, where only little net erosion was observed: Here it can be assumed that the net $\mathrm{C}$ deposition was also low and the statistical uncertainties can be determined by a statistical analysis of the deviation of the determined net $\mathrm{C}$ erosion from zero. The uncertainty for the net C erosion is smaller than the size of the data points in Fig. 6.

Due to the complete erosion of the $\mathrm{C}$ layer at the strike point position (see Fig. 5) only a lower value for the net carbon erosion can be determined directly from the measurements. In order to get a more realistic value for the net carbon erosion it was assumed that the shape of the carbon erosion is identical to the shape of the Mo erosion. This curve was then fitted to the measured C erosion close to the strike point position where the carbon was not fully eroded. This extrapolation of the carbon erosion based on the Mo erosion is shown in Fig. 6 as dash-dotted line. It should be noted that due to the different erosion mechanisms of carbon and molybdenum (carbon can be eroded by physical sputtering and by chemical erosion, while Mo is eroded only by physical sputtering by heavier impurities) this extrapolation is only valid for steady plasma and surface conditions throughout the whole campaign and therefore has large uncertainties. This is taken into account by assuming an uncertainty of $33 \%$ for the extrapolation: This range of uncertainties is shown as light grey area in Fig. 6 .

The maximum effective net carbon erosion rate at the strike line in standard configuration was in the range $5-8 \mathrm{~nm} / \mathrm{s}$.

The EBS results described above are in good agreement with the LIBS results from target element $1 \mathrm{u}$ TM2h6 [21]. Here also high erosion was observed at the strike line and almost the entire $C$ and Mo marker layer was erased in this area. Some redeposition of Mo on the uncoated bulk graphite area of these tiles was observed. Furthermore, lower $\mathrm{H}$ contents were observed around the strike line, which matches well with the heat flux pattern during plasma operation in standard configuration.

The eroded area ranges from about $-320 \mathrm{~mm}$ to $0 \mathrm{~mm}$ and is relatively wide. This is due to the movement of the strike line by toroidal currents [22]. In addition to the large erosion at the strike line at around $-90 \mathrm{~mm}$ a second line of enhanced erosion is observed at about $-210 \mathrm{~mm}$ : This is probably due to a second strike line which was already observed by thermography and appears at higher plasma currents [22].

For the vertical target element 5u TM2v2 the difference between the layer thicknesses before and after exposure, i.e. the net erosion of $\mathrm{C}$ and Mo, are shown in Fig. 7. See above for details of data processing and determination of uncertainties. As for the horizontal target elements the $\mathrm{C}$ layer was completely eroded from $-150 \mathrm{~mm}$ to $-70 \mathrm{~mm}$ and the real carbon erosion was extrapolated based on the Mo data (dash-dotted line in Fig. 7). 


\subsection{Total carbon erosion}

The toroidal distribution of carbon erosion integrated along the central line of target element TM2h6 is shown in Fig. 8 for all 10 TDU modules. The black bars indicate the directly measured C erosion. These values are, however, too low due to the complete erosion of the carbon marker at the strike line, see Figs. 5, 6 and 7. The grey bars indicate the carbon erosion based on the observed Mo erosion, see Figs. 6 and 7, the error bars take the uncertainty of this extrapolation (light grey area in Figs. 6 and 7) into account.

The difference in C erosion between the smallest erosion in $5 \mathrm{I}$ and the highest erosion in $2 \mathrm{u}$ is a factor of almost 2. The observed erosion pattern is in rough agreement with the energy deposition with symmetrized trim coil currents to different TDU target modules determined by thermocouples (TCS) on the rear side of the target modules (see Fig. 11 in [23]. Higher temperature rises of the TCs were observed for $2 u$ and $5 u$, lower temperature rises for $11,4 u, 51$ : This is in line with the observed net erosion pattern where higher erosion is observed in $2 u$ and $5 u$ and smaller erosion in $1 l, 4 u, 51$. But there are also some differences: The temperature rise was low in $3 l$ and $3 u$, while this is not visible as low erosion. These differences might be due to the fact that the erosion is integrated during the whole campaign while the TC temperature rise was only determined in single discharges. Moreover, the temperature rise of the TCs is proportional to the deposited energy, which in turn is approximately proportional to the gross carbon erosion. The measured net carbon erosion depends additionally on redeposition, which can be different in different TDUs for example due to locally different plasma parameters or impurity concentrations in the plasma, especially carbon and oxygen.

The erosion pattern varies not only between the different target modules in one TDU, but also between the different TDUs (see Fig. 8). According to the TC data from [23] TM1h to TM5h (see Fig. 2) receive the highest power fluxes in the horizontal targets during standard configuration discharges, while the fluxes to TM6h and TM7h are already considerably smaller. We therefore consider only carbon erosion from target modules TM1h to TM5 $\mathrm{h}$ for the calculation of the total carbon erosion, which means that the carbon erosion from 5 target modules in each of the 10 TDUs, i.e. in total 50 target modules in W7-X, has to be determined. Based on the thermographical images one can assume toroidal symmetry within each target module. Carbon erosion has been measured in poloidal direction on 14 horizontal target elements (see Table 2), which (based on the assumption of toroidal symmetry within each target module) can be extrapolated to 14 horizontal target modules. For the remaining 36 horizontal target modules the erosion can be extrapolated based on the TC data from [23] with symmetrized trim coil currents assuming that the carbon erosion is proportional to the temperature rise of the TCs. For all 10 horizontal targets this results in $34.5 \pm 8.4 \mathrm{~g}$ of net carbon erosion.

For the vertical targets the situation is more difficult: Each vertical target consists of 3 target modules, i.e. in total 30 target modules in W7-X. Only at 4 of these the poloidal distribution of erosion has been measured, see Table 2, and TC data have not been published. We therefore have to assume identical erosion on the 10 vertical targets of the TDU. Based on thermography images we assume that $50 \%$ of the toroidal length of TM $1 \mathrm{v}$ and $50 \%$ of the toroidal length of TM2v have full erosion and $50 \%$ of the toroidal length of TM2v have $50 \%$ erosion. With this assumption and the assumption of identical erosion in all 
TDUs, this gives a total carbon erosion at the vertical targets of $13.3 \pm 5.7 \mathrm{~g}$. The total carbon erosion from the horizontal and vertical targets of all 10 TDUs is therefore $48 \pm 14 \mathrm{~g}$ carbon.

\section{Discussion}

The PWI target elements should represent regular target elements as close as possible. As already reported for example in [Fehler! Textmarke nicht definiert.] the PWI target elements have a slightly different thermal capacity as regular TDU target elements. Moreover, some of the PWI target elements had leading edges. Both effects resulted in somewhat higher surface temperatures of the PWI target elements compared to regular target elements. While at the beginning of an experimental day all target elements had identical base temperature, the base temperature of the PWI target elements rose faster than the base temperature of regular elements throughout the day. The PWI target elements got also somewhat hotter by typically 50-100 K during plasma discharges. But it should be kept in mind that also some of the regular tiles had leading edges and got hotter than other tiles.

OP 1.2a was characterized by very high levels of $C$ and $O$ impurities in the plasma $[24,25]$. Carbon was therefore eroded by physical and chemical erosion by oxygen ions, physical sputtering by carbon ions, and physical and chemical erosion by hydrogen. It has been already observed at TEXTOR at conditions comparable to W7-X that for small plasma impurity concentrations the contributions of physical and chemical erosion of carbon by deuterium are almost identical [26]. However, for higher plasma impurity concentrations this may be different. The erosion yield of carbon by impact of oxygen ions is close to unity over a large range of energies [27] and temperatures from room temperature to above $1000 \mathrm{~K}$ [28]. The erosion is mainly due to chemical erosion by formation of $\mathrm{CO}$ and (to a minor degree) $\mathrm{CO}_{2}$ [28]. Physical sputtering plays a role only at energies above about $1 \mathrm{keV}$ [29], but remains always considerably smaller than chemical erosion. The physical sputtering yield of carbon by carbon ions with a few $100 \mathrm{eV}$ is 0.2-0.3 C/C-ion at normal incidence [27], but can get close to unity at grazing angles of incidence [29]. However, while self-sputtering of carbon can play an important role for the carbon concentration in the plasma, it is less relevant for the net erosion: Only sputtering yields above unity result in net erosion, smaller sputtering yields still result in net deposition of carbon. The total erosion yield of carbon by hydrogen ions is only 0.01-0.03 $\mathrm{C} / \mathrm{H}$-ion at temperatures between room temperature and $600 \mathrm{~K}$. At higher temperatures chemical erosion by formation of $\mathrm{CH}_{4}$ plays a more dominant role and the erosion yield can reach values of $0.1 \mathrm{C} / \mathrm{H}$-ion at $800 \mathrm{~K}$ [30]. The physical sputtering yield of carbon by helium ions is in the range $0.05-0.1 \mathrm{C} / \mathrm{He}$-ion $[27,29]$. With the high oxygen plasma concentrations in OP 1.2a the main erosion mechanism therefore was probably erosion of carbon by oxygen through formation of $\mathrm{CO}$. Physical sputtering of carbon by hydrogen is low and chemical erosion by formation of $\mathrm{CH}_{4}$ plays only a role at temperatures around $800 \mathrm{~K}$ : Such high temperatures were reached only for relatively short times in OP 1.2a. Nevertheless, chemical erosion can play a role due to the higher $\mathrm{H}$ fluxes. Further selfsputtering of carbon can maintain high carbon plasma concentrations, but, as discussed above, does not contribute to net carbon erosion. Physical sputtering by helium probably also plays only a minor role due to the relatively low helium concentrations in the plasma - at least compared to the oxygen concentrations. The quantification of the contributions of the different erosion mechanisms requires further investigations. 
Sputtering by oxygen (and potentially carbon) ions is also the most probable erosion mechanism for explaining the observed erosion of the Mo layers at the strike line: Sputtering of Mo by hydrogen-ions is very low with a threshold energy of $199 \mathrm{eV}$ [27]. Due to the low temperature dependence of carbon erosion by oxygen the somewhat higher temperature of the PWI target elements compared to regular target elements does not play a significant role.

In total almost $50 \mathrm{~g}$ carbon were eroded from the TDU in OP 1.2a, see section 3.3. To which places has this carbon been transported to? Carbon deposition on the TDU itself is low, see sections 3.1 and 3.2. This is a pronounced difference to tokamaks, where strong carbon deposition in the inner divertor or in remote divertor areas is observed $[31,32,33]$. Carbon-containing thin layers are observed at various places inside the main chamber of W7-X [34], but total amounts of deposited carbon are not yet available. Pump-out of carbon-containing molecules $\left(\mathrm{CO}, \mathrm{CO}_{2}, \mathrm{CH}_{4}\right)$ is also observed, but has not been quantified yet. Therefore, the carbon balance for $\mathrm{W} 7-\mathrm{X}$ requires further investigations.

\section{Conclusions}

The net erosion and deposition of carbon from the test divertor unit (TDU) of W7- $X$ was determined using special PWI target elements with marker layers consisting of about $300 \mathrm{~nm}$ Mo and 5-10 $\mu \mathrm{m} C$ on top during the operation phase OP 1.2a. The thicknesses of the marker layers were determined by elastic backscattering spectrometry (EBS) using $2.5 \mathrm{MeV}$ protons before and after exposure and laser-induced breakdown spectroscopy (LIBS) on selected target elements after exposure.

The erosion/deposition patterns determined by EBS and LIBS are in good agreement. Massive erosion of up to $20 \mu \mathrm{m}$ carbon was observed at the strike line. In total $48 \pm 14 \mathrm{~g}$ carbon were eroded from the TDU. The erosion was laterally non-uniform, strongly eroded surfaces were considerably smoother compared to the initial material. The most probable erosion mechanism is chemical erosion due to impact of oxygen ions and formation of $\mathrm{CO}$. Chemical erosion by hydrogen and formation of $\mathrm{CH}_{4}$ can also play a role, but only at elevated temperatures close to the maximum of chemical erosion. The quantification of the contributions of the different erosion mechanisms requires further investigations.

Only very little deposition of carbon is observed in remote areas of the TDU with little plasma contact. This is a profound difference to divertors in tokamaks, where eroded material is typically redeposited in remote divertor areas or in the inner divertor. The carbon balance for W7-X is not yet fully understood and requires further investigations. The hydrogen content of the TDU is relatively low and minimal at the strike point region as result of the elevated temperatures. The presented experimental results will be used as basis for modelling plasma-surface interaction processes in W7-X in order to gain deeper insight into the erosion mechanisms and material transport in $\mathrm{W} 7-\mathrm{X}$.

\section{Acknowledgements}


The measurements and spectrum evaluations of the tiles before exposure were performed by S. Bach. The technical assistance of J. Dorner and M. Fußeder from IPP Garching for their help with ion beam analysis measurements is gratefully acknowledged.

This work has been carried out within the framework of the EUROfusion Consortium and has received funding from the Euratom research and training programme 2014-2018 and 2019-2020 under grant agreement No 633053. The views and opinions expressed herein do not necessarily reflect those of the European Commission.

1 Beidler C et al. 1990 Physics and Engineering Design for Wendelstein VII-X Fusion Technol. 17148

2 Wolf R C et al. 2017 Major Results from the First Plasma Campaign of the Wendelstein 7-X Stellarator Nucl. Fusion 57102020

3 Bosch H-S et al. 2017 Final Integration, Commissioning and Start of the Wendelstein 7-X Stellarator Operation Nucl. Fusion 57116015

4 Sunn Pedersen T et al. 2017 Key Results from the First Plasma Operation Phase and Outlook for Future Performance in Wendelstein 7-X Phys. Plasmas 24055503

5 Peacock A et al. 2009 Progress in the Design and Development of a Test Divertor (TDU) for the Start of W7-X Operation Fusion Eng. Des. 841475

6 Sunn Pedersen T et al. 2019 First Results from Divertor Operation in Wendelstein 7-X Plasma Phys. Controlled Fusion 61014035

7 Sunn Pedersen T, Otte M, Lazerson S, Helander P, Bozhenkov S, Biedermann C, Klinger T, Wolf R C, Bosch H -S and the Wendelstein 7-X Team 2016 Confirmation of the Topology of the Wendelstein 7-X Magnetic Field to Better than 1:100,000 Nature Communications 713493

8 Hirsch M et al. 2008 Major Results from the Stellarator Wendelstein 7-AS Plasma Phys. Controlled Fusion 50 053001

9 Gao Y, Jakubowski M W, Drewelow P, Pisano F, Puig Sitjes A, Niemann H., Ali A, Cannas B and W7-X Team 2019 Methods for Quantitative Study of Divertor Heat Loads on W7-X Nucl. Fusion 59066007

10 Barbui T et al. 2018 The He/Ne beam diagnostic for active emission spectroscopy in the island divertor of Wendelstein 7-X, 45 ${ }^{\text {th }}$ EPS Conference on Plasma Physics, Prague, Czech Republic, P4.1018

${ }^{11}$ Effenberg $\mathrm{F}$ et al. 2019 Investigation of 3D effects on heat fluxes in performance-optimized island divertor configurations at Wendelstein 7-X Nucl. Mater. Energy 18262

12 Dhard C P et al. 2017 Preparation of Erosion and Deposition Investigations on Plasma Facing Components in Wendelstein 7-X Physica Scripta T170 014010

13 Greuner H, Boeswirth B, Boscary J and McNeely P 2007 High Heat Flux Facility GLADIS: Operational characteristics and results of W7-X pre-series target tests J. Nucl. Mater. 367-370 1444

14 Ruset C, Grigore E, Munteanu I, Maier H, Greuner H, Hopf C, Phylipps V and Matthews G 2009 Industrial Scale 10 $\mu \mathrm{mW}$ Coating of CFC Tiles for ITER-like Wall Project at JET Fusion Eng. Des. 841662

15 Dhard C P et al. 2019 Erosion and Deposition Investigations on Wendelstein 7-X First Wall Components for the First Operation Phase in Divertor Configuration Fusion Eng. Des. 146242

16 Mayer M 1997 SIMNRA User's Guide Technical Report IPP 9/113, Max-Planck-Institut für Plasmaphysik, Garching, Germany https://home.mpcdf.mpg.de/ mam/Report\%20IPP\%209-113.pdf 
17 Mayer M 2014 Improved Physics in SIMNRA 7 Nucl. Instrum. Methods Phys. Res. B 332176

18 Ziegler J F 2004 SRIM-2003 Nucl. Instrum. Methods Phys. Res. B 219-220 1027

19 Gurbich A F 2016 SigmaCalc Recent Development and Present Status of the Evaluated Cross-Sections for IBA Nucl. Instrum. Methods Phys. Res. B 37127

20 Mayer M 2002 Ion Beam Analysis of Rough Thin Films Nucl. Instrum. Methods Phys. Res. B 194177

21 Zhao D, Yi R, Oelmann J, Brezinsek S, Rasinski M, Mayer M, Dhard C P, Krause M and the W7-X team 2019 Ex-situ Analysis of W7-X Divertor Plasma-facing Components by Picosecond Laser Diagnostics Physica Scripta in print

22 Gao Y et al. 2019 Effects of Toroidal Plasma Current on Divertor Power Depositions on W7-X Nucl. Fusion submitted

23 Lazerson S A et al. 2018 Error Fields in the Wendelstein 7-X Stellarator Plasma Phys. Controlled Fusion 60124002

24 Sunn Pedersen T et al. 2018 First divertor physics studies in Wendelstein 7-X Proceedings of the Fusion Energy Conference 9

25 Wang E H et al. 2019 Impurity sources and fluxes in W7 X: from the plasma facing components to the edge layer Physica Scripta submitted

26 Brezinsek S et al. 2007 Hydrocarbon Injection for Quantification of Chemical Erosion Yields in Tokamaks J. Nucl.

Mater. 363-365 1119

27 Eckstein W, García-Rosales C, Roth J and Ottenberger W 1993 Sputtering Data Report IPP 9/82, Max-PlanckInstitut für Plasmaphysik, Garching, Germany http://hdl.handle.net/11858/00-001M-0000-0027-6324-6

28 Winter J 1996 Wall Conditioning in Fusion Devices and Its Influence on Plasma Performance Plasma Phys. Controlled Fusion 381503

29 Eckstein W 2002 Calculated Sputtering, Reflection and Range Values Report IPP 9/132, Max-Planck-Institut für Plasmaphysik, Garching, Germany http://hdl.handle.net/11858/00-001M-0000-0027-4522-5

${ }^{30}$ Balden M and Roth J 2000 New Weight-Loss Measurements of the Chemical Erosion Yields of Carbon Materials under Hydrogen Ion Bombardment J. Nucl. Mater. 28039

31 Mayer M, Rohde V, Ramos G, Vainonen-Ahlgren E, Likonen J, Herrmann A, Neu R and the ASDEX Upgrade Team 2007 The Deuterium Inventory in ASDEX Upgrade Nucl. Fusion 471607

32 Mayer $\mathrm{M}$ et al. 2009 Carbon Balance and Deuterium Inventory from a Carbon Dominated to a Full Tungsten ASDEX Upgrade J.Nucl. Mater. 390-391 538

33 Coad J P, Rubel M, Likonen J, Bekris N, Brezinsek S, Matthews G F, Mayer M and Widdowson A M 2019 Material Migration and Fuel Retention Studies during the JET Carbon Divertor Campaigns Fusion Eng. Des. 13878

34 Dhard C P et al. 2019 Inspection of W7-X Plasma Facing Components after the Operation Phase 1.2b:

observations and first assessments Physica Scripta submitted 
Table 1: Discharge statistics for OP 1.2a. A discharge is defined by a diamagnetic energy $\mathrm{W}_{\text {dia }}$ above $50 \mathrm{~kJ}$ or ECRH power above $0.1 \mathrm{MW}$ if $\mathrm{W}_{\text {dia }}$ is not available.

\begin{tabular}{|l|c|c|c|c|}
\hline Configuration & \multicolumn{2}{|c|}{ Number of discharges } & \multicolumn{2}{c|}{$\begin{array}{c}\text { Plasma time } \\
\text { (s) }\end{array}$} \\
\hline & H & He & H & He \\
\hline Standard & 236 & 421 & 655 & 1826 \\
\hline High lota & 27 & 117 & 55 & 379 \\
\hline High Mirror & 224 & 133 & 295 & 419 \\
\hline Unknown & 34 & 56 & 43 & 104 \\
\hline \multicolumn{1}{|c|}{ Total } & $\mathbf{1 2 4 8}$ & \multicolumn{2}{c|}{$\mathbf{3 7 7 6}$} \\
\hline
\end{tabular}


Table 2: Locations of all PWI target elements in the TDU, see Fig. 2 for a schematic graphical representation of the positions. TDU $1 \mathrm{l}$ is the lower TDU in W7-X module 1 , TDU $1 \mathrm{u}$ is the upper TDU in W7-X module 1 etc. TM2h6 is target module 2, horizontal, target element 6 . A similar nomenclature applies for all other target elements with $\mathrm{h}$ for horizontal and $\mathrm{v}$ for vertical modules.

\begin{tabular}{|l|c|}
\hline TDU & $\begin{array}{c}\text { Position of PWI } \\
\text { target element }\end{array}$ \\
\hline $1 \mathrm{l}$ & TM2h6 \\
\hline $1 \mathrm{u}$ & TM2h6 \\
\hline $2 \mathrm{l}$ & TM2h6 \\
\hline $2 \mathrm{u}$ & TM2h6 \\
\hline $3 \mathrm{I}$ & TM2h6 \\
\hline $3 \mathrm{u}$ & TM2h6 \\
\hline $4 \mathrm{I}$ & TM2h6 \\
\hline $4 \mathrm{u}$ & TM2h6 \\
\hline $5 \mathrm{I}$ & TM1h3 \\
& TM2h6 \\
& TM3h6 \\
& TM1v5 \\
& TM2v2 \\
\hline $5 \mathrm{u}$ & TM1h3 \\
& TM2h6 \\
& TM3h6 \\
& TM1v5 \\
& TM2v2 \\
\hline
\end{tabular}




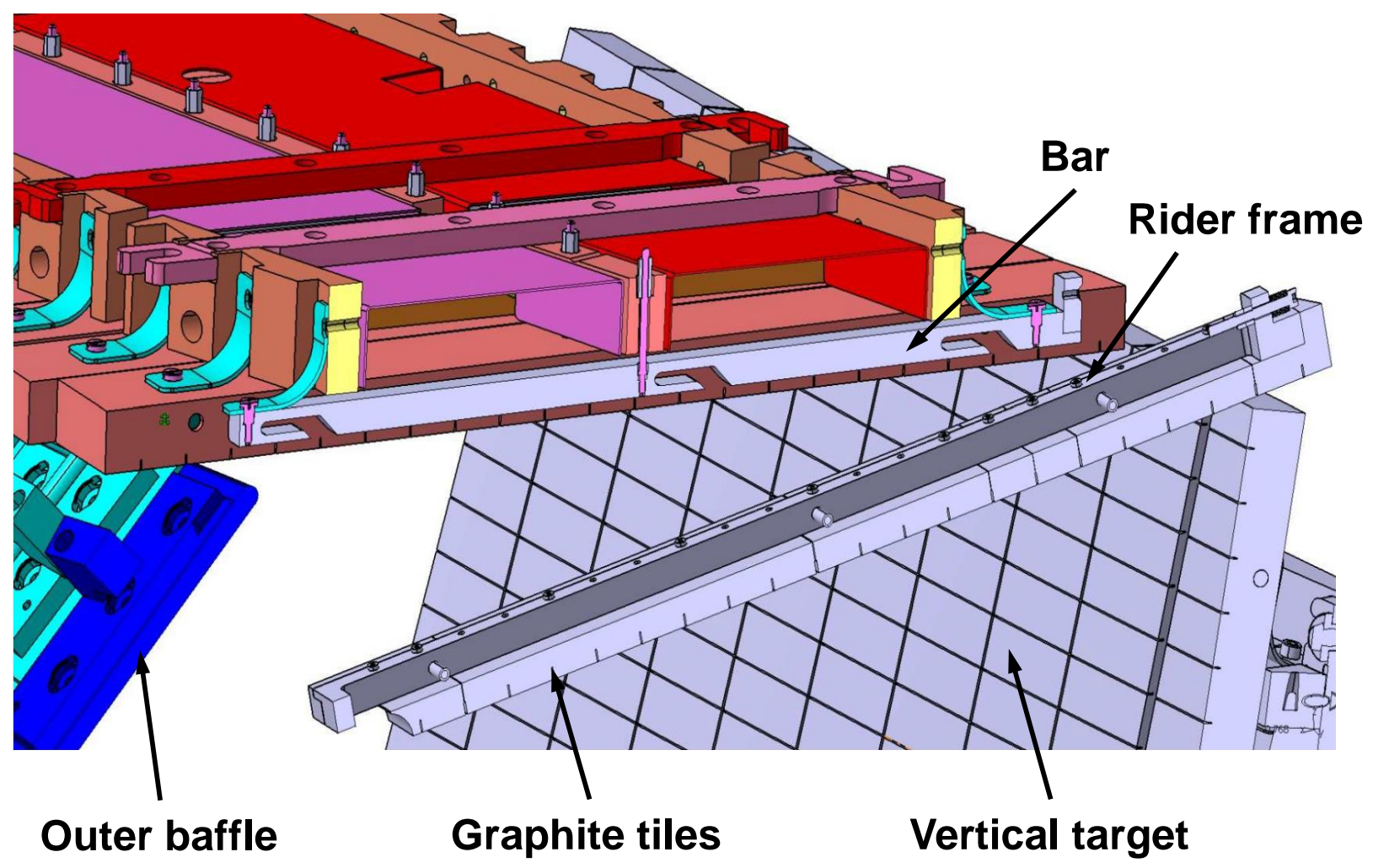

Figure 1: Schematic representation of the PWI target elements. 


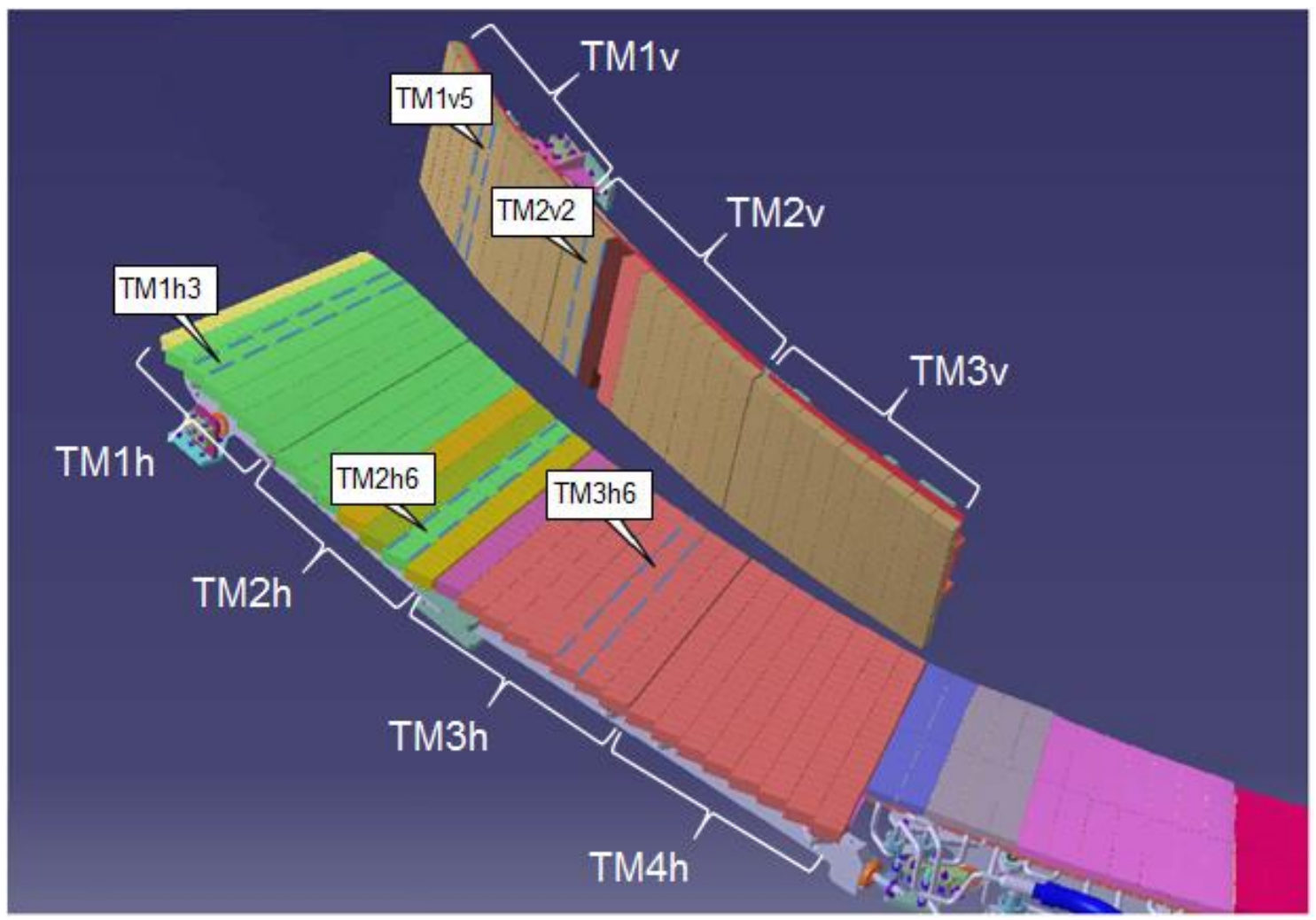

Figure 2: Schematic representation of the positions of the PWI target elements in the TDU. TMxh denotes horizontal target modules, TMyv are vertical target modules. See Table 2 for the nomenclature. 

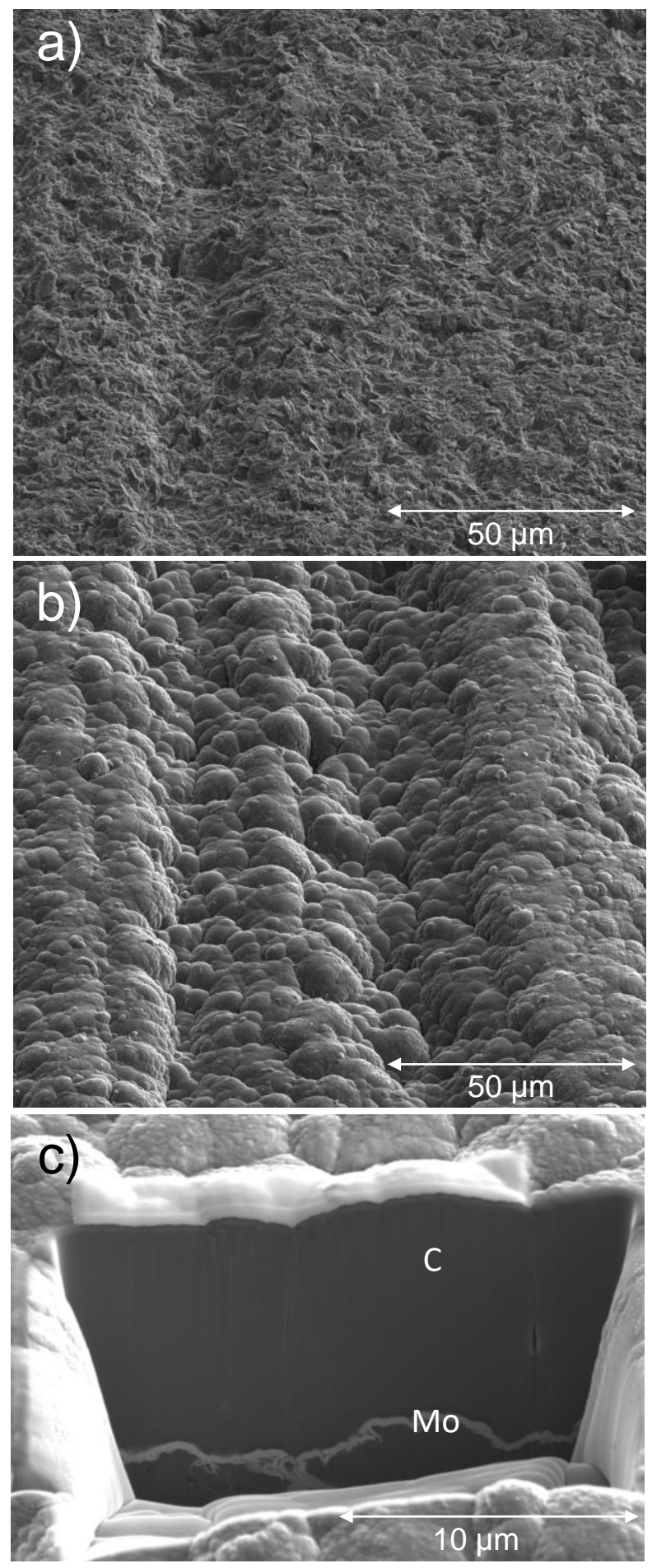

Figure 3: Scanning electron micrographs of unexposed samples. a) Fine grain graphite base material; b) $\mathrm{Mo} / \mathrm{C}$ marker coating. c) Focused ion beam cross-section of the $\mathrm{Mo} / \mathrm{C}$ marker coating. The inclination angle of the viewing direction is $52^{\circ}$ towards the surface normal. 

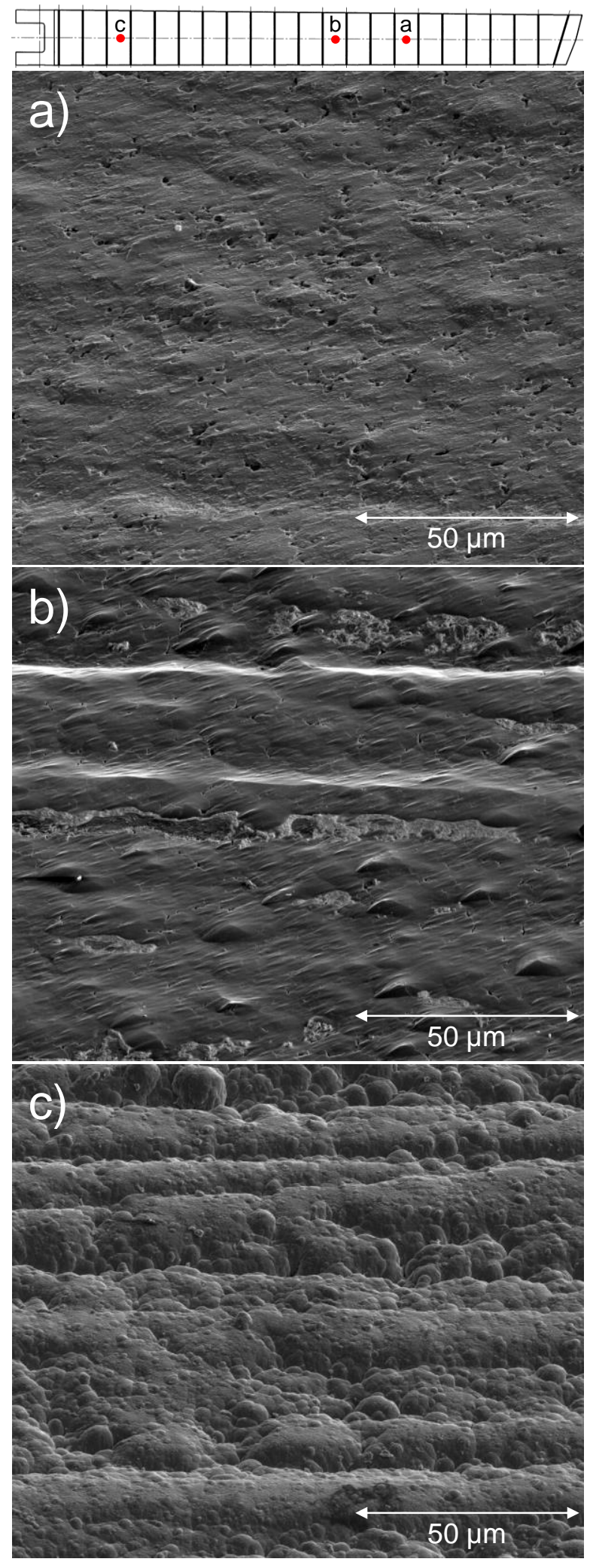

Figure 4: Scanning electron micrographs of the $\mathrm{Mo} / \mathrm{C}$ marker coating after exposure during OP 1.2a in TDU 2u TM2h6. a) high erosion at the strike point in standard configuration (center of tile \#120); b) medium erosion (center of tile \#121); c) remote area with thin deposits (center of tile \#124). The 
inclination angle of the viewing direction is $52^{\circ}$ towards the surface normal. A schematic representation of the target element is shown at the very top, positions of the micrographs are marked with dots. 


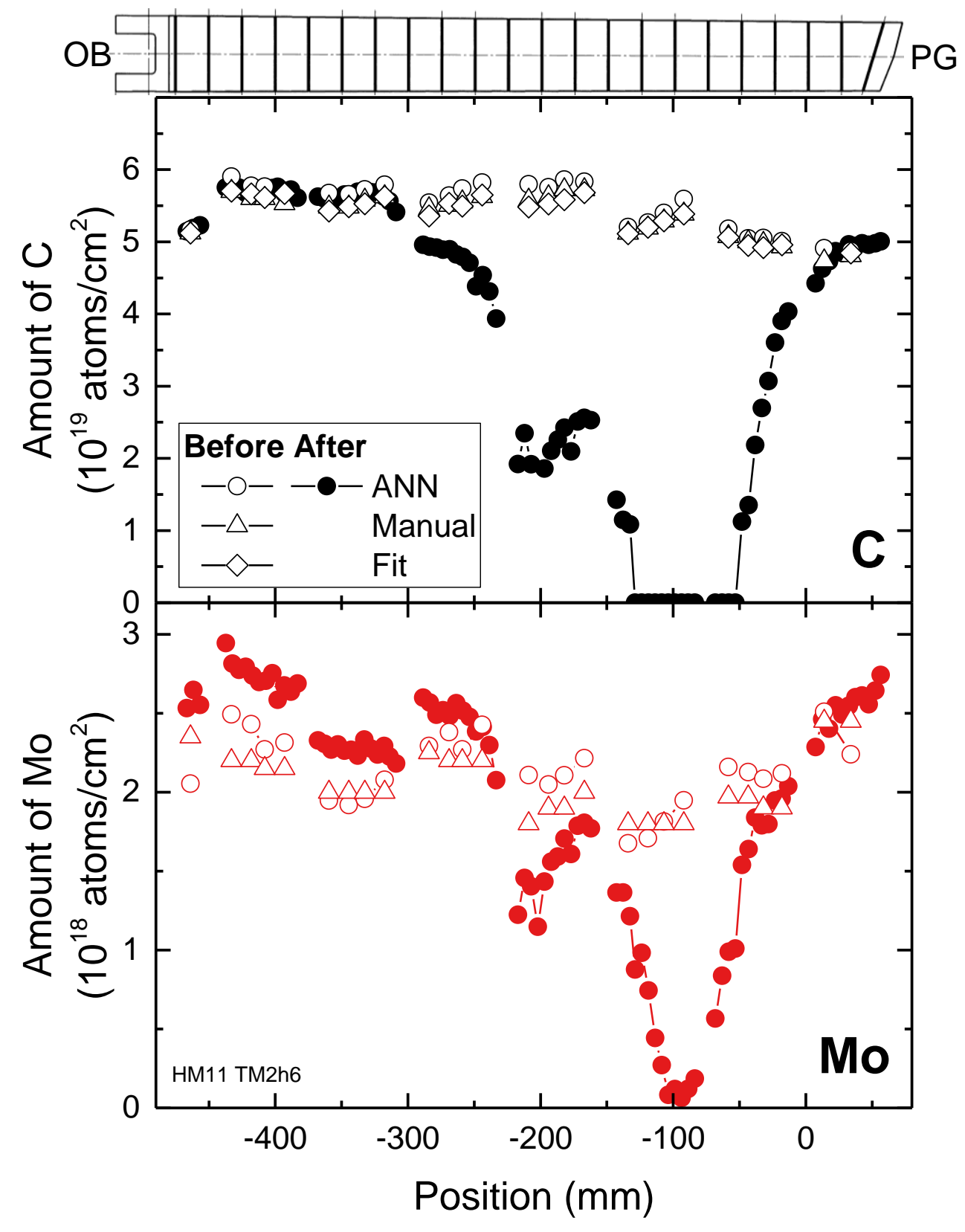

Figure 5: Thicknesses of the carbon (top) and molybdenum (bottom) marker layers before (hollow symbols) and after (solid symbols) exposure during OP 1.2a. Circles: thicknesses from the Artificial Neural Network (ANN) analysis; Triangles: thicknesses from manual fit; Squares: thicknesses from automatic fit. A schematic representation of the target element is shown at the very top. PG is the direction towards the pumping gap between the horizontal and the vertical targets, see Fig. 2, OB is the direction towards the outer baffle, see Fig. 1. TDU 1u TM2h6. 


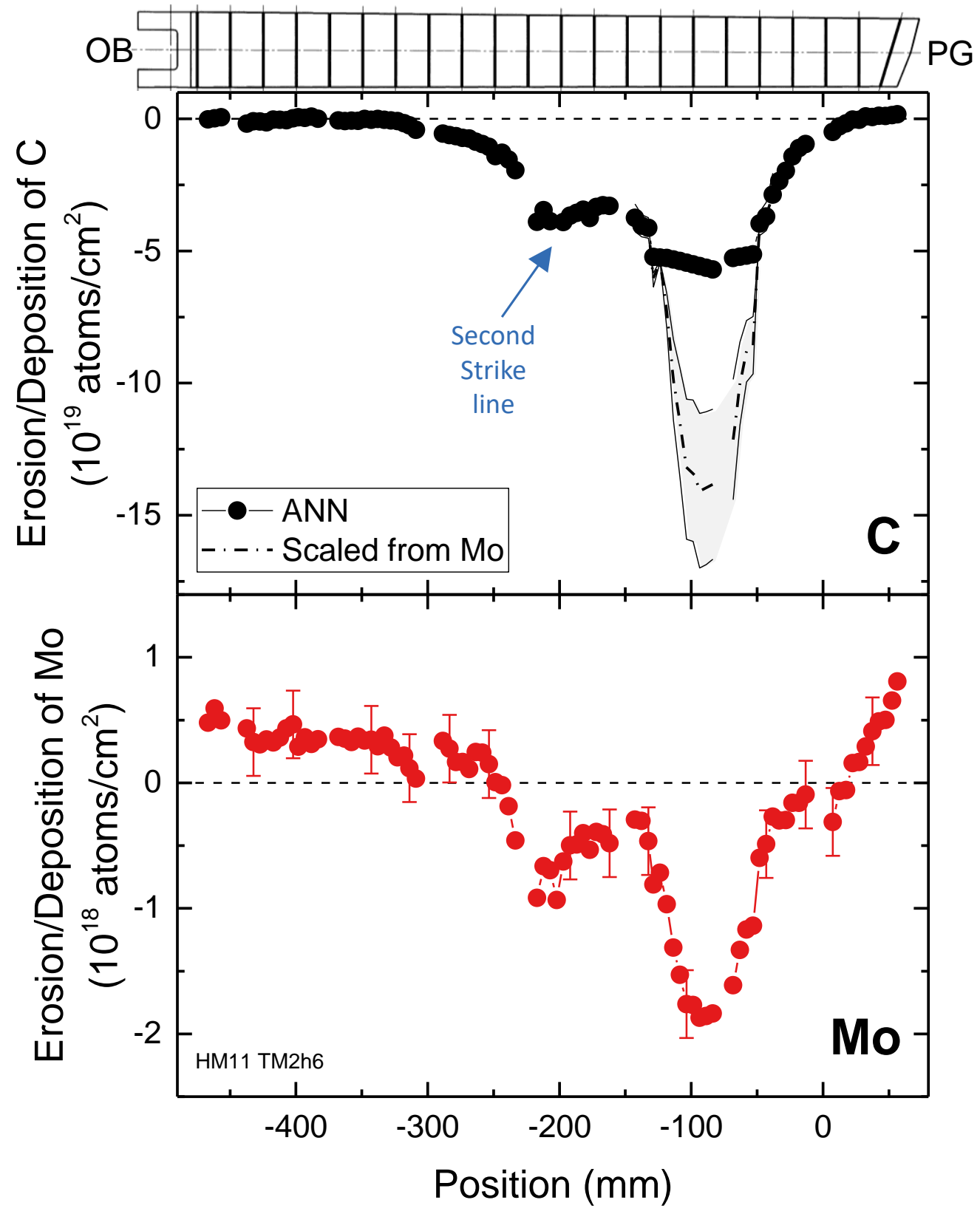

Figure 6: Net erosion of carbon (top) and molybdenum (bottom) from the marker layers during OP 1.2a based on the Artificial Neural Network (ANN) analysis. Deposition has positive, erosion negative sign. Dots: measured data; Dash-dotted line: extrapolated erosion of $\mathrm{C}$ based on the erosion of Mo; Grey area: uncertainty range of the extrapolated erosion of $C$. Typical experimental uncertainties are given for some points, the uncertainty for the $C$ erosion is smaller than the point sizes. A schematic representation of the target element is shown at the very top. PG is the direction towards the pumping gap between the horizontal and the vertical targets, see Fig. 2, OB is the direction towards the outer baffle, see Fig. 1. TDU 1u TM2h6. 


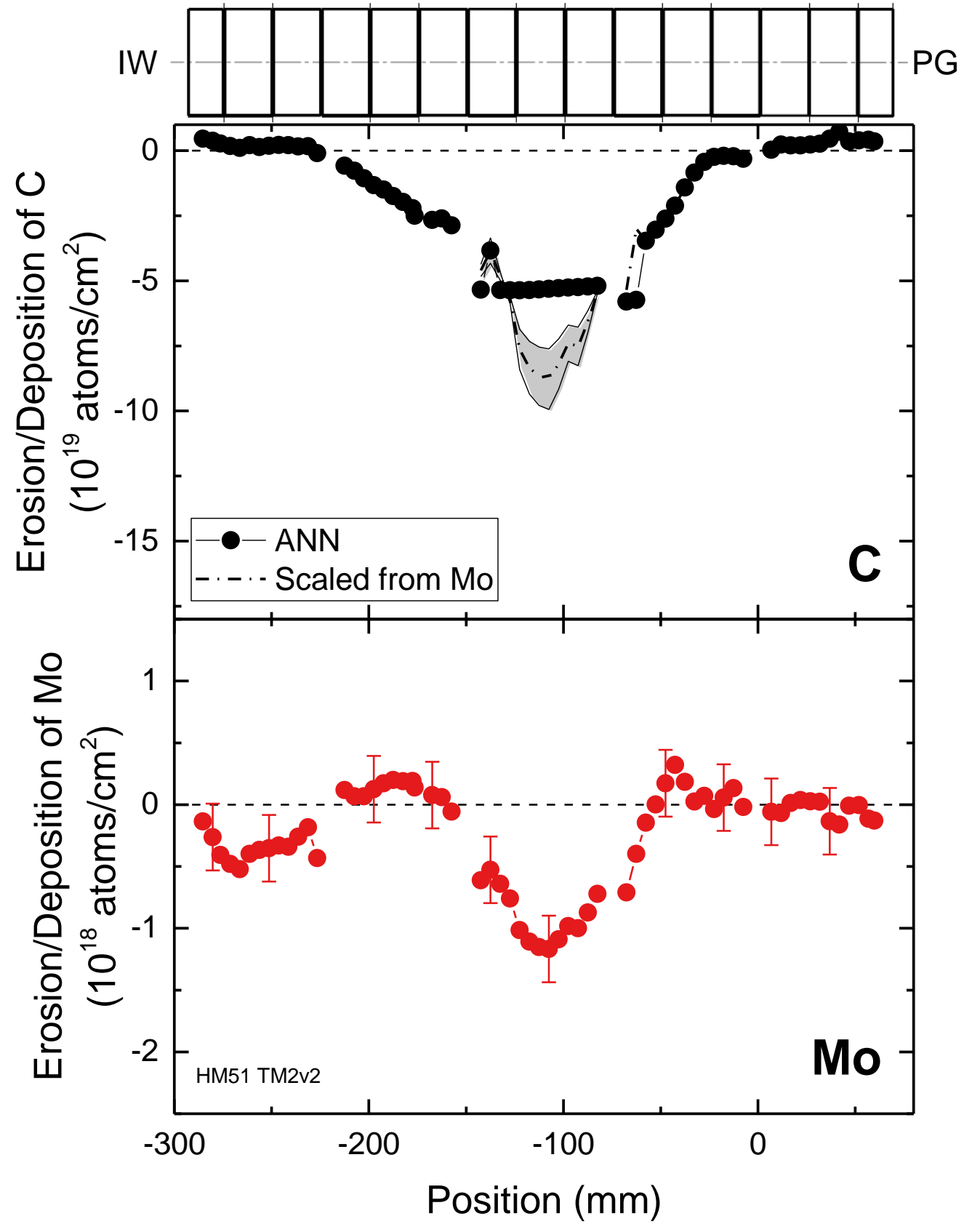

Figure 7: Erosion of carbon (top) and molybdenum (bottom) from the marker layers during OP 1.2a based on the Artificial Neural Network (ANN) analysis. Dots: measured data; Dash-dotted line: extrapolated erosion of $\mathrm{C}$ based on the erosion of Mo; Grey area: uncertainty range of the extrapolated erosion of $\mathrm{C}$. Typical experimental uncertainties are given for some points, the uncertainty for the $\mathrm{C}$ erosion is smaller than the point sizes. A schematic representation of the target element is shown at the very top. PG is the direction towards the pumping gap between the horizontal and the vertical targets, see Fig. 2, IW is the direction towards the inner wall. TDU 5u TM2v2. 


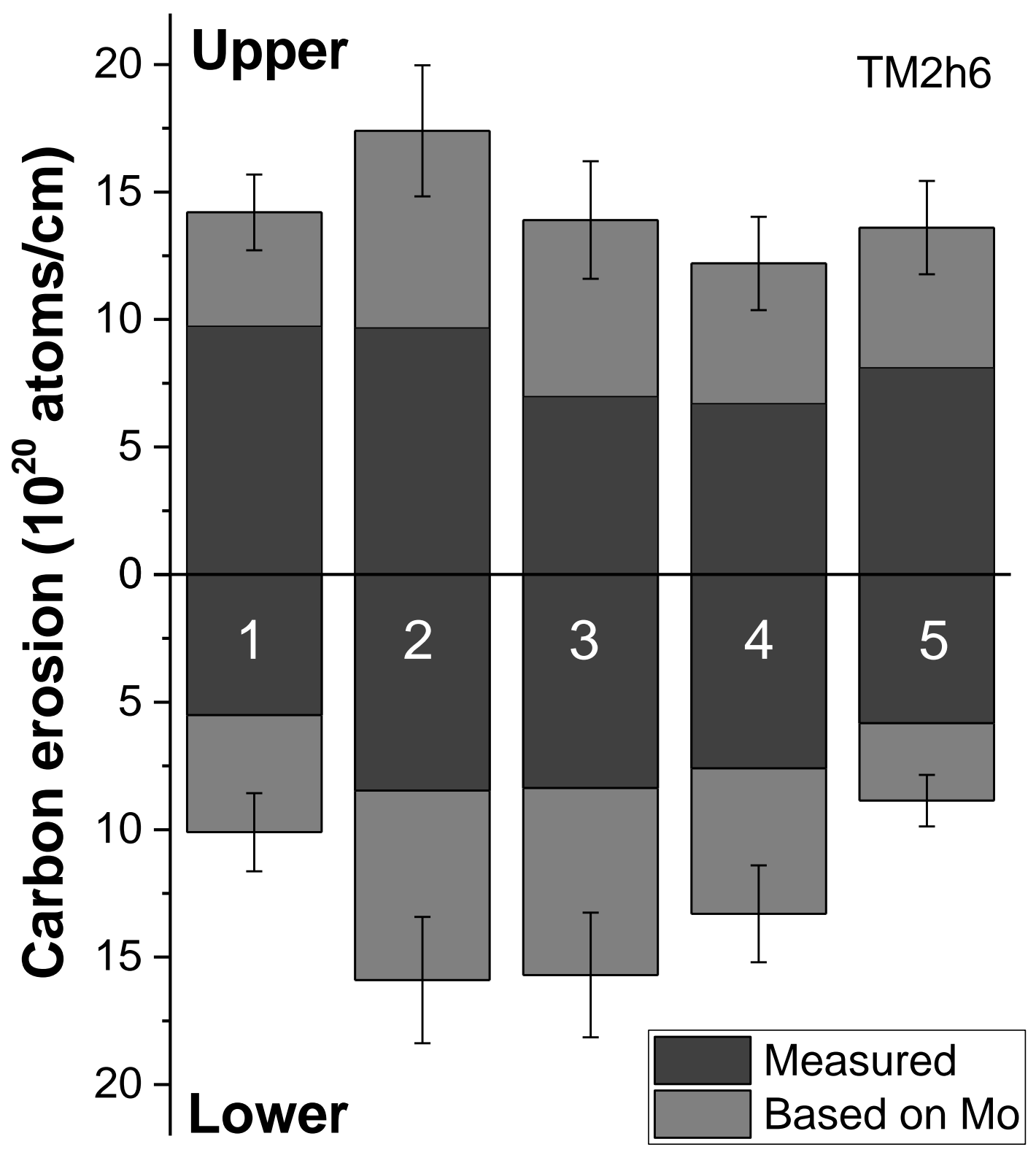

Figure 8: Erosion of carbon from the marker layers along the middle of target element TM $2 \mathrm{~h} 6$ in the upper and the lower TDUs in all 5 W7-X modules. Black bars: Measured carbon erosion; Grey bars: Carbon erosion extrapolated from the erosion of Mo, see Fig. 6. Uncertainties see Fig. 6. 